\title{
In Situ Growth of Metal Nanoparticles on Two-dimensional Materials Under Electrochemical Conditions
}

\author{
Shu Fen Tan, Kate Reidy, Haeyeon Lee and Frances Ross
}

Massachusetts Institute of Technology, Cambridge, Massachusetts, United States

The synthesis of metal nanoparticles (NPs) on two-dimensional (2D) materials opens up the possibility of novel functional composite nanostructures for many technological applications ranging from catalysis [1, 2] to nanoelectronic devices [3]. The interface between the metal NPs and the $2 \mathrm{D}$ material has a crucial role in determining the enhanced catalytic performance and tunable optical and electrical properties that can be achieved using metal-2D material heterostructures. Tremendous progress has been made in synthesizing ultrathin 2D materials decorated with metal NPs, in particular using vapour-phase deposition and wet chemical synthetic routes. Vapour-phase deposition methods such as thermal evaporation[4], atomic layer deposition[1] and electron-beam lithography[2] often require ultra-vacuum conditions and expensive equipment, resulting in low throughout and high cost of production. On the other hand, wet chemical synthetic routes are relatively easy to implement to produce NPs on 2D materials at a massive scale and under mild experimental conditions[5]. However, it can be difficult to tune the deposited morphology precisely in wet chemical synthesis involving the use of chemical reducing agents, due to preferred deposition at defect or surfaces with a large curvature[6]. Electrochemical deposition has evolved as a straightforward and versatile way to control the deposition of metal nanostructures[7]. Further improving the precision and application of electrochemical processing requires a deeper understanding of the interplay of kinetic and thermodynamic deposition phenomena such as NP shape, size and epitaxy and external parameters such as the thickness, nature and chemical moiety or identity of 2D materials, deposition potential and the temperature.

Here, we explore the possibility of using in situ liquid cell electron microscopy as a direct probe, with high temporal and spatial resolution, of the electrochemical deposition of noble metal NPs such as gold onto ultrathin 2D materials such as graphene and transition metal dichalcogenides in electrolytes containing aqueous metal precursors and hydrochloric or other acids. We transferred 2D materials onto liquid cell chips previously patterned with multiple metal electrodes and performed controlled electrochemical deposition by tuning the deposition potential as shown in Figure 1. Real-time observations reveal that the shape and size of the as-grown metal NPs are dependent on the nature of the 2D materials and metal NP growth with preferred orientation and alignment on 2D materials promoting epitaxy is anticipated. We will discuss the deposition mechanism, taking account of the effects of the electron beam on the materials and the deposition process, and discuss how the structures can be controlled via electrochemical and beam conditions (Figure 2). Understanding metal NP formation on 2D materials lays the foundation for rational design of functional materials for applications in opto-electronics, fuel-cells and catalysis. 


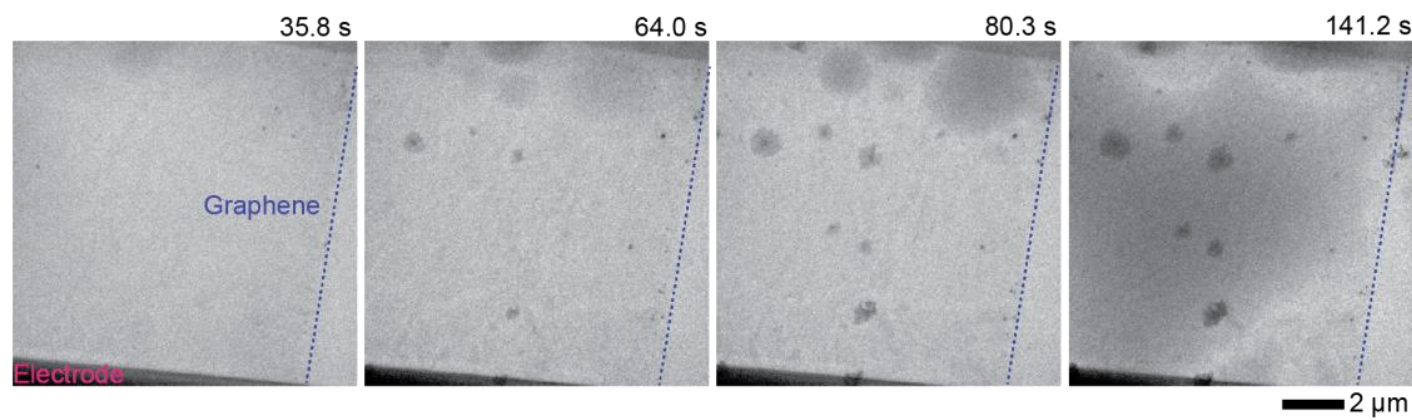

Figure 1. In situ electrochemical growth of Au nanoparticles on transferred graphene in acidic electrolyte solution. Time series of in situ TEM images showing the growth of Au nanoparticles on graphene (dotted blue lines) at working electrode during cyclic voltammetry from $0 \mathrm{~V}$ to $-1.5 \mathrm{~V}$ and back to $0 \mathrm{~V}$ at scan rate of $50 \mathrm{mV} / \mathrm{s}$ inside a liquid cell filled with $0.06 \mathrm{mM} \mathrm{HAuCl} 4+0.1 \mathrm{M} \mathrm{HCl}$ electrolyte solution at $23{ }^{\circ} \mathrm{C}$.
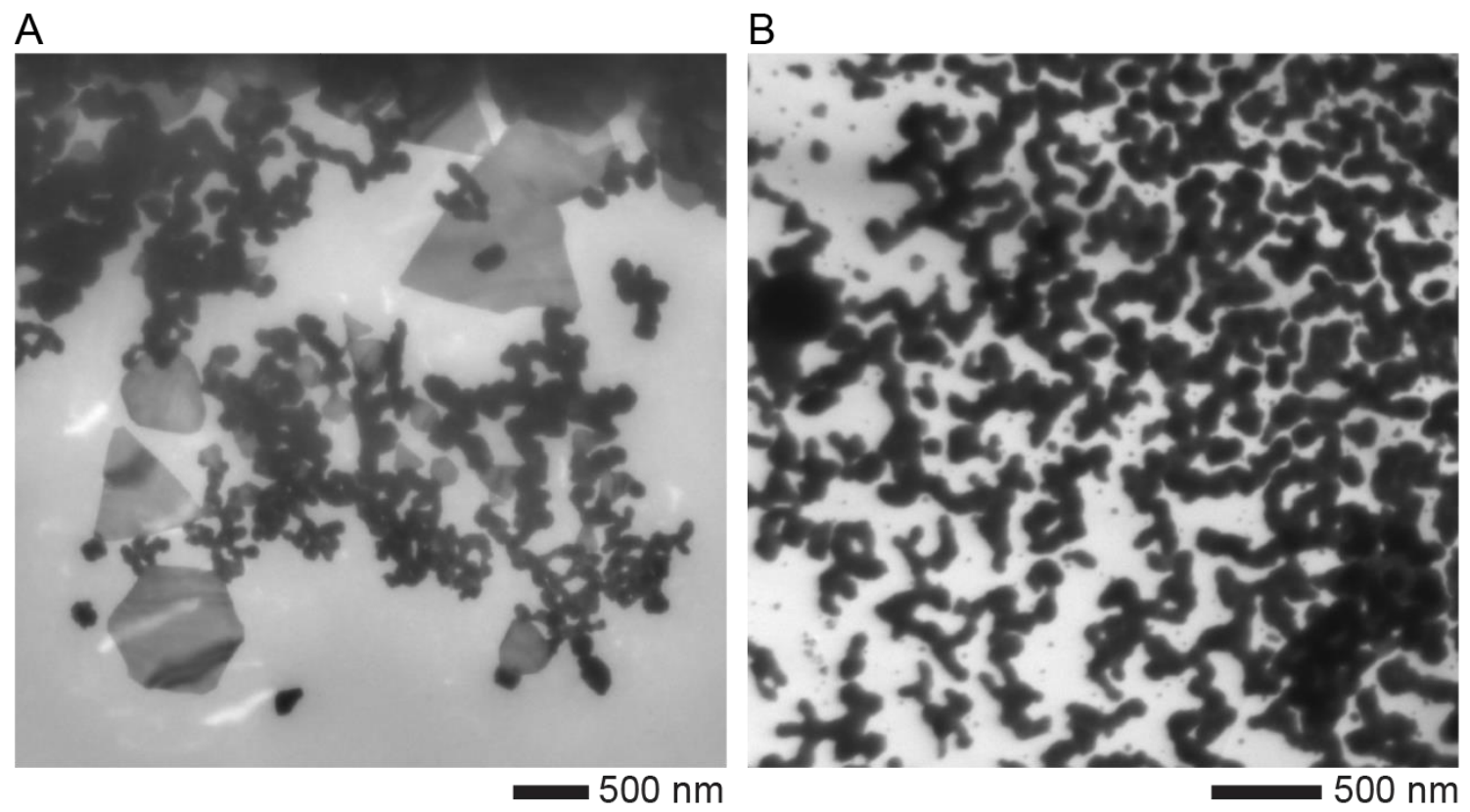

Figure 2. In situ electron beam-induced growth of $\mathrm{Au}$ nanoparticles on transferred (A) graphene (B) tungsten diselenide WSe2 in gold aurate precursor solution. TEM images showing the formation of $\mathrm{Au}$ nanoprisms on graphene while chain-like Au nanostructures were formed on WSe2 under electron beam irradiation.

References

1. Enterkin, J.A., K.R. Poeppelmeier, and L.D. Marks, Oriented Catalytic Platinum Nanoparticles on High Surface Area Strontium Titanate Nanocuboids. Nano Letters, 2011. 11(3): p. 993-997.

2. Komanicky, V., et al., Shape-Dependent Activity of Platinum Array Catalyst. Journal of the American Chemical Society, 2009. 131(16): p. 5732-5733.

3. Huang, Y., et al., Directed Assembly of One-Dimensional Nanostructures into Functional Networks. Science, 2001. 291(5504): p. 630-633.

4. Yoo, Y., et al., Steering Epitaxial Alignment of $A u, P d$, and AuPd Nanowire Arrays by Atom Flux Change. Nano Letters, 2010. 10(2): p. 432-438. 
5. Huang, X., et al., Solution-phase epitaxial growth of noble metal nanostructures on dispersible singlelayer molybdenum disulfide nanosheets. Nature Communications, 2013. 4(1): p. 1444.

6. Figuerola, A., et al., Epitaxial CdSe-Au Nanocrystal Heterostructures by Thermal Annealing. Nano Letters, 2010. 10(8): p. 3028-3036.

7. Yang, H., Platinum-Based Electrocatalysts with Core-Shell Nanostructures. Angewandte Chemie International Edition, 2011. 50(12): p. 2674-2676. 